\title{
Corela
}

Cognition, représentation, langage

HS-32 | 2020

Les postures énonciatives

\section{Éthos discursif, éthos préalable et postures énonciatives}

Ethos discursif, éthos préalable et postures énonciatives

\section{Ruggero Druetta et Paola Paissa}

\section{(Q) OpenEdition}

\section{Journals}

Édition électronique

URL : https://journals.openedition.org/corela/12457

DOI : $10.4000 /$ corela. 12457

ISSN : 1638-573X

\section{Éditeur}

Cercle linguistique du Centre et de l'Ouest - CerLICO

\section{Référence électronique}

Ruggero Druetta et Paola Paissa, «Éthos discursif, éthos préalable et postures énonciatives 》, Corela [En ligne], HS-32 | 2020, mis en ligne le 09 novembre 2020, consulté le 13 juillet 2021. URL : http:// journals.openedition.org/corela/12457 ; DOI : https://doi.org/10.4000/corela.12457

Ce document a été généré automatiquement le 13 juillet 2021.

\section{(c) (1) (9)}

Corela - cognition, représentation, langage est mis à disposition selon les termes de la licence Creative Commons Attribution - Pas d'Utilisation Commerciale - Partage dans les Mêmes Conditions 4.0 International. 


\title{
Éthos discursif, éthos préalable et postures énonciatives
}

\author{
Ethos discursif, éthos préalable et postures énonciatives
}

Ruggero Druetta et Paola Paissa

1 Le questionnement sur la relation des postures énonciatives avec l'éthos (recouvrement des notions ou complémentarité ; liens possibles entre construction de l'éthos et choix de telle ou telle posture) représentant l'un des axes majeurs de ce recueil, nous entendons nous interroger sur la nature de ce rapport. Si Rabatel, à plusieurs reprises, et notamment dans la citation suivante, a déjà souligné une différence fondamentale dans le statut des notions :

"Il faut aussi distinguer les postures de l'éthos, qui est à l'origine une image de soi
argumentant, dont on étend le domaine de validité à tous les phénomènes
langagiers (Amossy 2010) : là encore, les postures sont limitées à la co-construction
des PDV, et la "présentation de soi" ne concerne la posture que pour autant qu'elle
entre dans cette construction. " (Rabatel $2017: 78$ )

2 Nous comptons pour notre part, dans un premier temps, prolonger cette réflexion sur le plan théorique et, dans un deuxième temps, illustrer, sur le plan applicatif, des configurations dans lesquelles se manifeste la participation des postures à la construction de l'éthos. Avant de considérer quelques études de cas montrant la dynamique des deux notions, nous allons d'abord, ci-dessous, comparer leur empan.

\section{Postures énonciatives et éthos : l'empan des deux notions}

3 La notion d'éthos et celle de posture énonciative partagent un certain nombre de traits, qui se laissent ramener aux deux points suivants :

a) Les deux notions relèvent du domaine du caractère, des attitudes et des comportements (individuels et collectifs) susceptibles d'observation dans l'espace discursif et, plus largement, dans l'espace social ${ }^{1}$. Cela signifie que les deux concepts entretiennent à la fois, du point de vue linguistique, des relations avec les notions d'empathie, de position et de positionnement aux sens définis par Rabatel 2012a et 
2017 ; du point de vue social, des rapports avec certains acquis sociologiques (cf. les concepts de champ, d'habitus, d'hexis au sens bourdieusien).

b) Les deux notions présentent, en même temps, une nature évolutive et projective. Évolutive, puisque tant l'éthos que les postures énonciatives sont susceptibles de se transformer dans le temps et de s'afficher de manière plus ou moins intense (régime de "scalarité » des postures : cf. Rabatel 2012a et 2017 ; manière plus ou moins explicite d'exprimer l'éthos dit ou l'éthos montré: cf. Maingueneau 2002). Projective, puisque toutes deux, bien qu'à des degrés différents, sont envisageables en tant que résultats d'une co-construction, d'une négociation avec les attentes et les prévisions $\mathrm{du} / \mathrm{des}$ destinataires (suivant un processus d'interprétation pour les postures et d'incorporation pour l'éthos).

Cependant, l'empan des deux notions présente à l'évidence une grande différence d'ampleur, ainsi que des spécificités épistémologiques et fonctionnelles que nous allons énumérer brièvement ci-dessous :

a') la notion d'éthos appartient à la rhétorique argumentative et la notion de posture énonciative concerne la linguistique de l'énonciation. Comme les travaux d'Amossy et de Maingueneau l'ont montré de manière convaincante, cette distinction épistémologique n'implique pas l'indépendance de l'éthos vis-à-vis de l'énonciation. Cependant, c'est d'une façon différente que l'éthos relève de l'énonciation: alors que l'éthos peut préexister à l'énonciation (éthos préalable²) et s'avérer englobant (suivant Maingueneau, il « enveloppe » l'énonciation, sans besoin d'être explicité, pouvant être montré et non dit $\left.^{3}\right)$, la posture énonciative ne peut qu'être saisie dans l'hic et nunc du discours proféré : elle représente donc une entité segmentable, discrétisable du dit. Sujettes à des micro-variations ponctuelles, les postures peuvent néanmoins articuler, autant que l'éthos, le verbal et le coverbal.

b') L'hétérogénéité foncière et le caractère innombrable des marques est une caractéristique commune tant à l'éthos qu'aux postures énonciatives. Toutefois - et c'est là une conséquence du point précédent - la typologie des marques ayant été jusqu'ici mises en valeur est fort inégale. Si, pour les marques de posture, on liste en général des marques linguistiques et/ou discursives, telles que le lexique, la syntaxe, la prosodie et éventuellement "la dimension coverbale» (encore que cette dernière "dimension» ait été jusqu'ici moins étudiée que d'autres), les marques éthotiques peuvent intégrer, de surcroît, des éléments extra-verbaux ${ }^{4}$ (l'habillement, la fréquence des apparitions publiques, l'adhésion à des initiatives humanitaires, etc. : cf. Rabatel $2005 \mathrm{~b}: 102$ ), la notion d'éthos empiétant, par ce biais, sur celle de « style $»^{5}$.

7 c') La dimension dialogique est un trait intrinsèque et définitoire des postures énonciatives. De même, l'éthos est façonné par l'interdiscours et construit dans et par le discours, un éthos pouvant soit s'accommoder des attentes de l'auditoire (cf. l'éthos de Hollande dans le discours du Bourget dans Rabatel, à paraitre), soit se dresser en opposition à un éthos hétéroattribué et refusé, dans un mouvement de retournement du stigmate ${ }^{6}$. Cependant, le dialogisme caractérisant l'éthos ne participe pas, de la même manière que les postures énonciatives, d'un travail de communication finalisé à une entente réciproque. Bien que la possibilité existe d'étudier les variations de postures dans le désaccord (Rabatel 2017 : 93), la notion de posture a été conçue dans le cadre d'une conception finalement coopérative du langage (au sens gricéen). En effet, au sein de cette conception, la taxinomie des postures (et non seulement la coénonciation, au demeurant assez rare et fuyante : Rabatel 2004 et 2005 a) est conçue 
comme un dispositif destiné, lors de la co-construction des PDV, à nuancer les formes de l'accord ou, du moins, à tempérer les conflits en cas de positionnements dissensuels.

d') Enfin, une différence de taille concerne le(s) sujet(s) au(x)quel(s) les deux notions peuvent s'appliquer. Alors que les postures ne concernent que l'énonciateur (en tant que source d'un PDV, pouvant coïncider avec le locuteur de l'énoncé, L/E, ou être « interne » à l'énonciation, e1 ou e2), l'éthos peut être l'affaire tant de l'énonciateur (dont, éventuellement, on "prend en compte» le dire ${ }^{7}$ ) que du locuteur. Dans ce dernier cas, l'éthos peut en outre se rapporter soit au locuteur L (être de discours) soit au locuteur $\lambda$ (être du monde), donnant lieu à des relations de convergence ou de divergence entre l'éthos discursif et l'éthos préalable. De plus, selon qu'il touche à l'énonciateur ou au locuteur et selon les formes qu'il peut assumer, l'éthos relève du régime de l'énonciation explicite (éthos dit), du régime de la monstration (éthos montré) ou encore de la représentation (éthos représenté).

\section{Postures énonciatives et éthos : quelques figures d'une complémentarité}

9 Une disproportion existe donc entre le nombre limité des postures formant la topique énonciative et l'éventail bien plus vaste des manifestations éthotiques, ce qui rend impossible, dans l'espace d'un article, de saisir toutes les configurations dans lesquelles s'articulent ces deux facteurs. Cependant, force est de constater que la posture adoptée par l'énonciateur, dans une situation discursive de co-construction d'un PDV, contribue, en association avec un faisceau d'autres traits, à l'élaboration de son éthos. Posant l'hypothèse d'une complémentarité des deux éléments, nous allons notamment nous interroger :

1) sur la possibilité d'identifier des correspondances systématiques entre telle posture et tel type d'éthos, tout en rappelant que les régularités observées jusqu'ici en matière de postures énonciatives sont rares (on peut citer les corrélations concernant certains dispositifs figuraux, notamment l'hyperbole ou l'ironie et la sur-énonciation ; l'humour et la sous-énonciation décrits par Rabatel 2012b, 2013 et 2014, ou des correspondances de type générique, comme celles que décèle Florea 2015 entre la sur-énonciation et le blâme ; la sous-énonciation et l'éloge, etc.) ;

2) sur la possibilité de considérer les postures comme des marques de l'éthos, s'ajoutant à la liste indéfinie de celles-ci ${ }^{8}$;

3) sur l'articulation du plan de l'énonciation et des marques posturales avec le plan de l'argumentation et de l'effet pragmatique que l'énonciateur vise, par l'adoption de telle posture et de tel éthos.

Pour mettre en évidence le rôle de l'éthos et la synergie qui se crée entre sa construction et la posture adoptée, nous avons décidé de choisir des situations de « crise éthotique » ou de « retravail de l'éthos » (Amossy 2010 : 89-93), c'est-à-dire des conjonctures dans lesquelles l'image de l'énonciateur est mise en cause, celui-ci s'évertuant à la défendre, voire à la rehausser. Dans la première partie, nous prendrons en considération, à travers le cas de Nicolas Hulot, le jeu des postures énonciatives dans la recherche d'un équilibre entre l'éthos préalable et l'éthos discursif, relativement aux trois formes de l'éthos (dit, montré, représenté). Dans la deuxième, nous nous pencherons, en revanche, sur le cas d'Emmanuel Macron, en nous concentrant sur la dialectique des reprises et des reformulations (auto- et hétéro-dialogiques) et sur le rôle de la scénographie énonciative (Maingueneau 2002). 


\subsection{Nicolas Hulot entre éthos d'activiste et éthos de ministre}

11 Toute l'expérience gouvernementale de Nicolas Hulot a vu le conflit de deux ethè : d'un côté, son éthos préalable d'écolo ultra-populaire et, de l'autre, son nouvel éthos de ministre d'État. Or, c'est, entre autres choses, par le jeu des variations des postures énonciatives que ce conflit a été géré, à la fois par Hulot lui-même (éthos dit et montré) et par les différents acteurs politiques ou médiatiques qui ont commenté ses actions (éthos représenté).

12 Nous limitant à la toute dernière phase de l'expérience politique de Hulot, nous observerons : pour ce qui est de l'éthos dit et montré, une situation dialogale (quelques extraits de l'annonce de sa démission surprise : interview du 28 août 2018 sur France Inter) ainsi qu'un discours monogéré (discours de passation des pouvoirs du 4 septembre 2018); pour l'éthos représenté, quelques commentaires qui ont suivi ces événements.

13 L'interview où Hulot prend tout le monde de court, en annonçant son départ, entrecroise constamment l'argument ad humanitatem, qui se ramène à l'éthos de l'activiste soucieux de l'avenir de la planète, à l'argument ad personam, qui réagit aux attaques et défend la politique des « petits pas " accomplis par le gouvernement, envers lequel Hulot, dans une posture de co-énonciation, déclare avoir " une immense amitié » et auquel il « (s)'excuse de faire une mauvaise manière ».

Or, deux passages de l'interview, se référant aux mesures concrètes mises en place pendant l'année du ministère Hulot, sont indicatifs de la solidarité qui s'établit entre posture et éthos. Le premier précède l'annonce de la démission et représente une réponse piquée à la question de Léa Salamé de savoir si dans la deuxième année de ministère « on en restera encore aux belles paroles ${ }^{9}$ :

(1) NH : Il n'y a pas que les belles paroles + il y a quand même de l'action il y a eu de l'action dans l'année et contrairement à ce qu'on dit + la France en fait plus que beaucoup de pays + ne me faites pas dire qu'elle en fait assez + elle n'en fait pas assez l'Europe n'en fait pas assez le monde n'en fait pas assez.

Dans cette réplique, Hulot accomplit des ajustements rapides de ses postures et de son éthos. Dans un premier moment, il réagit en défendant, malgré tout, son image de ministre (ce qui est cohérent avec l'attitude de co-énonciation qu'il emprunte en général envers le gouvernement): il adopte une posture de sur-énonciation (" contrairement à ce qu'on dit »), qui lui permet d'afficher également une certaine fierté nationale, suivant l'éthos collectif, quelque peu clichéique, de la "République exemplaire ». Cependant, l'éthos de l'écologiste déçu prend tout de suite le dessus, s'accompagnant d'abord d'une posture momentanée de sous-énonciation (« ne me faites pas dire que... ») pour laisser la place, successivement, au ton péremptoire de la sur-énonciation, dans les reprises symétriques, assenées sur un rythme martelant « elle n'en fait pas assez, l'Europe n'en fait pas assez, le monde n'en fait pas assez ».

Le deuxième passage contredit partiellement ce que Hulot a affirmé dans le passage précédent et à d'autres moments de l'interview. Bien qu'il assume, pendant toute l'interview, un éthos montré de souffrance intérieure et un éthos dit de franchise absolue (à plusieurs reprises, il assure, malgré son amertume, vouloir être sincère, cesser de se mentir, etc.), lorsque Nicolas Demorand lance sur BFMTV la déclaration de 
Benjamin Griveaux, le porte-parole du gouvernement, qui évoque des «petits pas réalisés ", Hulot s'exclame :

(2) $\mathrm{NH}$ : Je ne veux pas polémiquer et et une $\mathrm{f}$ - et + et je vous $\mathrm{d}$ - après je + m'effacerai ++ eh mais j'en profite + les petits pas ++ mais mais + vous croyez que + la situation climatique + s'accommode des petits pas + vous croyez que l'état de la planète s'accommode des petits pas vous croyez que les inégalités euh qui s'exposent dans un monde qui aujourd'hui est connecté s'accommodent des petits pas + mais ça fait 40 ans qu'on s'accommode des petits pas et c'est pour ça qu'on est dans une situation qui nous dépasse ++ et souvent, on me disait + «t'es content on a fait ce que tu as demandé »++ mais, moi, ce n'est pas ça que j'avais envie d'entendre +++ je n'avais pas envie d'entendre qu'on fasse des choses pour me faire plaisir $+\mathrm{j}$ 'avais envie d'entendre des ch- + qu'on faisait des choses parce qu'on avait compris la gravité de la situation.

À l'attitude de co-énonciation envers le gouvernement ( «Je ne veux pas polémiquer et après, je m'effacerai ») succèdent ici : 1) la reprise d'une posture de sur-énonciation, liée cette fois-ci à l'éthos de l'activiste qui prend la journaliste (et l'auditoire) à témoin d'une contradiction criante et la re-proposition de la stratégie rhétorique de la répétition pressante («vous croyez que.... ? », réitéré trois fois);2) la caricature de la posture de co-énonciation qu'ont adoptée envers Hulot les autres membres du gouvernement pendant l'année de son ministère. En effet, dans une sorte de microrécit, Hulot représente, sous une forme quasiment parodique, l'éthos de bienveillance et l'attitude de co-énonciation de ses collègues qui, pendant une année, ont essayé de le ménager (« et souvent, on me disait, etc. »). Comme à d'autres moments de l'interview (cf. plus haut : «On me dit mais prends ton temps ++ sois patient ++ mais ça fait trente ans qu'on est patient »), c'est le connecteur oppositif « mais » qui indique une rupture à plusieurs niveaux: i) suivant sa fonction canonique, le connecteur marque une inversion argumentative; ii) en même temps, il marque un virage brusque, à la fois dans la gestion des plans temporels (l'année de son ministère vs une durée indéfinie, en tout cas trop longue : 30 ans, 40 ans) et dans la gestion des postures et de l'éthos. Face à la marque énonciative « on » qui, de manière ambivalente, indique ici tant l'humanité qui subit le manque d'action politique, que le gouvernement dont Hulot a fait partie, s'affrontent deux ethè individuels : celui de l'activiste en sur-énonciation, qui récupère son idiolecte "groupal» (Rabatel 2005 b et $2017: 247$ ) : «mais ça fait 40 ans qu'on s'accommode des petits pas... ») et celui du ministre récalcitrant, quasiment infantilisé, en sous-énonciation ( $"++$ mais, moi, ce n'est pas ça que j'avais envie d'entendre +++ je n'avais pas envie d'entendre qu'on fasse des choses pour me faire plaisir + j'avais envie d'entendre des ch- + qu'on faisait des choses parce qu'on avait compris la gravité de la situation »).

Alors que l'interview (situation dialogale) où il annonce son départ présente aussi quelques moments de sous-énonciation où Hulot, à l'instigation des journalistes à qui il s'adresse, affiche un éthos de modestie, sans doute de circonstance (il assume la responsabilité de sa faillite et admet de n'avoir « probablement " pas su se montrer à la hauteur de la situation), le discours de passation des pouvoirs (situation monologale) fait triompher de nouveau la sur-énonciation et l'éthos de l'écologiste hyper-engagé, porteur d'espoir. En dépit d'un éthos montré d'idéaliste déçu (l'émotion, intensément montrée, provoque ses larmes et l'oblige à interrompre son allocution), dans le discours du 4 septembre, Hulot affiche de nouveau une complémentarité forte entre la posture de sur-énonciation et l'éthos de l'activiste, se montrant optimiste, voire prophétique. En effet, à plusieurs reprises il a recours à des formes d'incitation collective : 
" puissions-nous : ++ méditer cette phrase ${ }^{10}$ » « je sais intimement que partout + dans ce pays ++ il y a dans l'ombre un souffle puissant d'humanisme + de créativité + puissionsnous collectivement l'entendre l'accompagner l'encourager le multiplier "; « osons la solidarité [...] osons l'espoir + osons l'utopie »; «soyons disruptifs soyons inventifs + soyons + créatifs ".

Quant à l'éthos représenté de Nicolas Hulot, qui se dégage des différents commentaires à sa démission, la gamme des possibilités est fort vaste et s'accompagne généralement d'une posture de sur-énonciation. En effet, il s'agit de commentaires qui portent moins sur son geste ${ }^{11}$ que sur Hulot en tant qu'«être du monde » et qui juxtaposent incessamment le plan de l'évaluation de ses traits psychologiques individuels (un homme " fragile ", « immature ", « trop perfectionniste ", etc.) à celui de l'appréciation politique ("un écologiste autoritaire», un «trouillard», un "paravent du gouvernement ${ }^{12}$ ", etc.). Une position plus nuancée et plus intéressante, pour montrer la complémentarité de l'éthos et des postures, est représentée par l'attitude adoptée par le Président Macron. En effet celui-ci, en reconnaissant que Nicolas Hulot est « un homme libre » et en soulignant de l'avoir choisi justement pour cela, tente le coup de force de faire coexister une posture de co-énonciation et un éthos de bienveillance ( Je respecte sa liberté et je souhaite pouvoir compter sur l'engagement de Hulot, sous une autre forme et là où il sera »), avec une attitude de sur-énonciation et un éthos de volonté (« si j'ai choisi Nicolas Hulot il y a quinze mois, c'est parce qu'il est, entre autres, un homme libre»). Le Président tente ainsi de bloquer la dérive des commentaires quelquefois médisants des différents ministres envers Hulot $^{13}$ et de donner le coup d'envoi à un discours d'éloge de la politique environnementale du gouvernement qui, « en quinze mois a fait plus qu'aucun autre sur ce sujet ${ }^{14}$.

C'est justement le cas d'Emmanuel Macron - un Président qui tente constamment de louvoyer entre des tendances opposées ${ }^{15}$ - qui nous occupera dans la deuxième partie, consacrée à approfondir la question de l'écart se creusant parfois entre les postures affichées par des marques ostensibles et l'intention pragmatique (perlocutoire) que l'on peut prêter à l'énonciateur.

\subsection{Postures et éthos dans l'interview télévisée d'Emmanuel Macron du 15 octobre 2017}

21 Pour analyser de manière extensive les phénomènes d'éthos dit et montré ainsi que leur articulation éventuelle à la gestion des postures énonciatives, nous avons choisi la première interview qu'E. Macron a accordée à un groupe de journalistes le 15 octobre 2017, dans son bureau à l'Élysée. Nous nous sommes tout spécialement intéressés au discours rapporté comme manifestation marquée du dialogisme constitutif de l'énonciation. Dans ce cadre, nous avons pris en compte les aspects verbaux de ce marquage, ainsi que l'intertextualité avec des prises de parole précédentes redoublement autodialogique pour le locuteur présidentiel, dédoublement hétérodialogique pour les journalistes qui évoquent ces paroles et invitent le président de la République à revenir sur celles-ci afin de les évaluer, de s'en expliquer et/ou de se corriger. Par ailleurs, nous avons également souhaité analyser l'un des nombreux discours en réaction à cette interview, pour l'étude des postures du locuteur citant dans une argumentation visant à stigmatiser l'éthos du Président par le truchement d'une 
posture de sur-énonciation surplombante et comportant la sélection, dans le discours rapporté, d'un certain nombre de segments posant problème.

C'est en effet sur un questionnement pressant autour de l'éthos du président que s'ouvre l'interview du 15 octobre, suite à de nombreux écarts verbaux repris par la presse et stigmatisés par une large partie de l'opinion publique et des opposants politiques. La reprise de ces mots est associée à une posture de sous-énonciation, à mettre peut-être en relation avec le respect pour la fonction présidentielle. Les journalistes accompagnent les citations d'expressions multiples d'excuses, qui limitent la prise en charge à leur compte :

(3) J1 : monsieur le président vous parlez de vos discours de vos déclarations + (il)

faudrait qu'on s'attarde un instant sur votre vocabulaire les Français vous ont entendu prononcer les mots " cynique " «fainéants " à d'autres moments ils vous ont entendu pardon pour les oreilles chastes dénoncer ceux qui «foutent le bordel » est-ce qu'un président peut parler comme ça est-ce qu'un chef de l'état peut de temps à autre s'exprimer comme Michel Audiard?

(4) J3 : pardon de revenir au au terme « bordel » pardon

L'emploi du conditionnel par J1 (il faudrait qu'on s'attarde) et la multiplication des précautions (pardon, employé par J1 et J3) semblent viser pragmatiquement la préservation de la face positive du Président ${ }^{16}$, qui risque d'être ternie par le rappel de ce vocabulaire à la source d'une crise éthotique.

Dans un autre passage de l'interview (5), le discours rapporté par les journalistes est celui des ministres d'Emmanuel Macron. La posture est, une fois de plus, de sousénonciation car, dans leur stratégie de questionnement, ils effectuent une quasi-PEC et veulent que ce soit le Président qui prenne sur lui la responsabilité de la qualification évaluative, à travers la structure alternative de la question (sélection ou pas sélection?), destinée à empêcher toute réponse élusive :

(5) J3 : vous osez parler de sélection + ou c'est encore tabou

EM : mais il y a une sélection aujourd'hui + c'est le tirage au sort + vous avez suivi comme moi l'actualité + et il y a une sélection.

J2 : Et vos ministres parlent ils ont beaucoup de précautions oratoires ils parlent de prérequis + sélection ou pas sélection

Les journalistes ne sont pas pour autant dans une attitude de complaisance vis-à-vis de leur interlocuteur et, après ce début précautionneux, peuvent prendre à l'occasion une posture de sur-énonciation, voire de dissensus, que ce soit à travers la question rhétorique (comment avez-vous pu dire... ?) de (6) ou les caractérisations axiologiques (clivant, stigmatisant) de (7) :

(6) J1 : Mais comment avez-vous pu + dire je rencontre parfois dans une gare + des gens qui ne sont rien ça vous a échappé ou

EM : Non parce que parce que je n'ai pas dit cela + non + je n'ai pas dit cela

$\mathrm{J} 1$ une gare c'est un lieu où on croise des gens qui réussissent et des gens et des gens qui ne sont rien

(7) EM : les mots de «cynique » et de «fainéant » sont d'un registre ++ normal ou soutenu

$\mathrm{J} 1$ : mais clivant mais stigmatisant + non?

Dans d'autres cas, lorsqu'aucun enjeu éthotique n'est directement sollicité, la posture de co-énonciation peut se manifester dans le discours des journalistes. Ainsi, lorsque Macron s'exprime à propos de l'ISF et des mesures prises par Hollande, le journaliste abonde dans ce sens par une citation, que le président approuve d'un hochement de tête, avant de terminer son argumentation en (8). De même, en (9), le discours rapporté 
du journaliste, au beau milieu de l'argumentation d'Emmanuel Macron, semble destiné à lui tendre une perche dont celui-ci se saisit par une auto-reprise en co-énonciation (L1/E1/e1, redoublement autodialogique) :

(8) EM : il y a cinq ans mon prédécesseur a augmenté massivement + l'ISF il a créé une taxe + à $75 \%$ + pour + celles et ceux qui gagnaient + plus d'un million

$\mathrm{J} 1$ : vous aviez dit vous-même que c'était Cuba sans le soleil +++ oui

$\mathrm{EM}$ : est-ce que ça a rapporté beaucoup beaucoup d'argent on disait les chiffres allaient être mirifiques non pourquoi?

(9) EM : il faut qu'on s'assure qu'il recherche bien de manière active un emploi donc il faut vérifier euh + les efforts qui sont faits et il faut vérifier que + c'est pas un multirécidiviste du refus donc je ne veux plus qu'il y ait d'effets d'aubaine

L1 : combien de + combien de refus + cinq ou six c'est important

$\mathrm{EM}:$ ça ils devront en définir les modalités

L4 : vous aviez dit deux vous aviez dit deux

EM : moi j'avais dit deux je pense que c'est sain + mais je pense que ce contrôle ça doit être un contrôle + bah de l'engagement de celui qui est en recherche d'emploi + de cette bonne diligence + et un contrôle qu'on ait face à nous quelqu'un qui ne refuse pas $\mathrm{X}$ fois

En ce qui concerne le discours du Président, en revanche, c'est surtout la surénonciation qui domine, dans deux types de discours rapporté différents : la citation des propos d'un auteur connu ou fictif et le discours rapporté d'un interlocuteur fictif. Dans le premier cas (10), la citation concerne le principe d'effectivité de la philosophe Simone Weil, que l'auteur avait déjà citée dans son discours au Congrès, le 3 juillet $2017^{17}$ : le régime est celui de la sur-énonciation parce que non seulement le locuteur ne retient que le radical du mot, qui est utilisé ici avec une terminaison adjectivale, mais encore parce qu'il le soustrait au discours d'origine pour le plier au sens - tout-à-fait ordinaire - de sa propre argumentation dans le contexte des mesures concernant l'accès des bacheliers à l'université. La fonction de la citation est d'ennoblir les propos du locuteur par le contact avec une philosophe emblématique du XX $\mathrm{XX}^{\mathrm{e}}$ siècle et de leur donner ainsi plus d'efficacité : il s'agit en somme d'un argument d'autorité à travers une citation d'autorité, où c'est davantage le nom de l'auteur, plutôt que son verbatim, qui réalise l'effet pragmatique recherché.

(10) EM : et donc vous le voyez + juste pour conclure ce point + je veux que + en même temps qu'on libère + on protège + et l'esprit de ces réformes c'est bien + ce couple libérer protéger et les garanties effectives pour reprendre + le beau mot de de la philosophe Simone Weil + c'est non pas d'aller expliquer aux gens qu'ils ont des droits des droits de tirage des droits dont ils ne verront jamais la couleur + c'est de leur dire + la société change je ne vais pas vous prop- vous proposer de vous protéger contre la société ou son changement + je vais vous prop-poser de vous( armer pour trouver une place dans cette société qui change

On retrouve un procédé semblable, toujours en sur-énonciation, mais avec une posture feinte de sous-énonciation, pour les citations des jugements du dictionnaire de l'Académie. Le contexte dans lequel ces citations surgissent est celui de la réponse aux critiques sur ses "éléments de langage ", jugés blessants et arrogants. La stratégie argumentative consiste à départager la cause en ayant recours à une autorité tierce. La soumission à la parole du Dictionnaire permet donc d'avoir un argument d'autorité pour forcer également les journalistes et l'opinion publique à s'y soumettre : c'est l'autorité qui parle, il faut s'incliner. Du point de vue énonciatif, le locuteur est certes dans une posture apparente de sous-énonciation, mais, pragmatiquement, cette posture lui permet de se ranger derrière le PDV de l'autorité pour forcer - perlocutoirement - 
l'accord argumentatif. Le locuteur s'installe donc dans une attitude dominante vis-à-vis de ses destinataires ${ }^{18}$.

(11) EM : les mots de «cynique » et de " fainéant » sont d'un registre ++ normal ou soutenu [...] le mot « bordel » c'est du registre + populaire + comme dit + l'Académie française + populaire

La sur-énonciation est en revanche la posture ouvertement adoptée par le locuteur dans le cas de discours représentés fictifs mettant en scène des énonciateurs appartenant à telle catégorie visée par l'argumentation. Dans les exemples (12) et (13), il s'agit des «jaloux » vis-à-vis des "premiers de cordée », dont Macron rapporte les propos et qu'il qualifie de "pas cohérents» (12), tandis qu'en ce qui concerne leurs énonciateurs, « il leur manque quelque chose dans le raisonnement » (13) :

(12) EM : on ne peut pas dire d'un côté + «c'est( affreux il y a des entreprises qui sont achetées par des groupes internationaux il n'y a plus de capital en France regardez + nos groupes sont( ++ pris par l' international » et de l'autre dire «je déteste les gens qui ont du capital quand bien même ils l'investissent en France »++ ça ça n'est pas cohérent

(13) EM : ceux qui vous disent d'un côté + « je suis pour un ISF massif + je ne veux pas que les gens soient riches dans mon pays » et qui de l'autre vous disent « je suis pour la souveraineté industrielle» il leur manque quelque chose dans le raisonnement

Dans le deuxième cas (14), Emmanuel Macron brosse la situation qu'il envisage pour traiter les travailleurs mis à pied dans le contexte des restructurations, dans un dialogue fictif entre Pôle Emploi et les partenaires sociaux. Le locuteur citant est en coénonciation avec l'énonciateur dont les propos sont rapportés (L1/E1/e2), puisque cela correspond à ses vœux, ce qui est marqué par le modal déontique devront :

(14) EM : les partenaires sociaux devront nous éclairer sur ce point en disant + « il ne faut pas que ce soit un moins de tant de pourcentage de son dernier salaire faut pas que ce soit trop loin ou si c'est trop loin faut le compenser "

Ce qui se dégage de l'analyse énonciative du rapport des instances de prise en charge impliquées dans cette interview (tout particulièrement dans le cas du discours rapporté), c'est que, malgré le caractère momentané et local (Rabatel 2017) - donc, somme toute, "pulvérisé » des postures - les locuteurs semblent être en adéquation avec ce qu'on peut considérer comme les postures prototypiques ${ }^{19}$ des journalistes et du responsable politique: la sous-énonciation comme cadre général pour les journalistes ${ }^{20}$, avec des incursions en sur-énonciation (mais toujours subordonnées à une attitude de déférence et de politesse); la sur-énonciation pour le responsable politique, comme marque d'une autorité dont la parole est régulatrice, et qui ne doit se soumettre qu'à l'autorité des grands esprits, quitte à les récupérer pour ses propres finalités argumentatives, opérant ainsi un basculement de la sous-énonciation apparente (pour reprendre le beau mot de la philosophe Simone Weil) à la sur-énonciation effective (c'est non pas de... mais de...) d'un locuteur-roi qui a le pouvoir de re-nommer la réalité et, à travers cette parole que la loi rend démiurgique, de la transformer.

\section{3. Éthos préalable, éthos dit et montré. Contribution des postures énonciatives}

Les aspects énonciatifs que nous venons de présenter sont susceptibles d'être mis au service de la stratégie pragmatique d'argumentation du locuteur en tant qu'éléments 
ponctuels de l'éthos montré, car ils participent d'une mise en scène de l'énonciateur en train d'organiser son discours. Comme l'éthos fait feu de tout bois, il n'est pas étonnant que les postures aussi fassent l'objet d'une récupération dans la construction active de l'éthos du locuteur. Le président « jupitérien », à travers ses postures (feintes) de sousénonciation, montre ainsi son attitude respectueuse vis-à-vis des autorités de la langue, sorte de limitation symbolique de son pouvoir absolu, tout en s'assimilant en même temps à elles pour en partager finalement le pouvoir normatif. Par ses postures de surénonciation, par contre, il manifeste pleinement son rôle et sa fonction. À ce titre, il est utile de se pencher sur les commentaires métadiscursifs donnés comme réponses aux critiques concernant le «style " présidentiel ${ }^{21}$ et encadrant les fragments citationnels présentés dans le paragraphe précédent. Il s'agit dans ce cas d'éthos dit, qui interagit avec l'éthos montré au niveau de la gestion énonciative et des postures du locuteur, s'opposant à un éthos préalable que les journalistes n'ont de cesse de rappeler.

En réaction à une crise éthotique, déclenchée par quelques écarts verbaux prononcés à plusieurs occasions ponctuelles et amplifiés par le débat politique et les médias, Macron déploie une stratégie basée essentiellement sur la preuve éthique et sollicitant tout particulièrement les dimensions de l'areté et de l'eunoia, tandis que la phronesis ne semble pas avoir été spécialement mise à contribution, sauf au tout début de l'interview, lorsqu'il proclame sa volonté « de ne pas avoir une présidence bavarde », de vouloir garder une certaine forme de retenue et de "solennité ». À travers cette déclaration initiale, il se met en scène comme détenteur d'une certaine forme de sagesse (phronesis) et vise à capter la bienveillance des spectateurs. Il s'agit, à proprement parler, de la captatio benevolentiae de la rhétorique classique, placée à l'exorde d'un discours. Macron choisit donc une ouverture canonique pour commencer sa défense.

Dans la suite de l'interview, étant donné que les critiques portent sur son style et son langage, sa stratégie argumentative portera en revanche davantage sur les dimensions de l'areté et de l'eunoia. En effet, face à l'accusation d'utiliser un langage dysphorique et vulgaire, en inadéquation avec la fonction présidentielle qu'il recouvre, Macron choisit de ne pas nier mais, après avoir allégué l'autorité des dictionnaires et rappelé le caractère privé de ses mots (" c'était dans un aparté »), il argumente qu'il s'agit là de sa franchise, de son courage à nommer les choses (areté). Il utilise d'ailleurs ce verbe de façon absolue à plusieurs reprises, sans adverbe de modalisation ( «je nomme, je nomme les choses ») : il nomme les choses par leur nom, il nomme vrai, donc pas besoin d'autres mots qui réduiraient la portée de cette action ontologiquement irréprochable. L'abondance de la première personne prouve bien que nous avons affaire ici à un exemple d'éthos dit :

(15) EM : mais je réponds très clairement d'abord +++ j'ai toujours + essayé de dire les choses + depuis que je suis engagé dans la vie politique et de m'approcher + d'une forme de vérité + celle que je pensais + juste + et donc je nomme + je l'ai fait il y a plusieurs années lorsque j'ai parlé + de l'illettrisme je l'ai fait à plusieurs reprises y compris durant la campagne +++ nos élites politiques se sont habituées à ne plus dire les choses + à avoir un discours + en quelque sorte aseptisé et + à considérer que + ce qui était intolérable c était le mot que l'on mettait et pas la réalité qu'il y avait derrière non

(16) EM : mais je s- je continuerai à dire les choses je continuerai à respirer l'air + à parler à nos concitoyens à parfois + m'emporter quand j'échange avec eux et à dire + ce que je crois vrai

(17) EM : mais derrière + je ne suis pas dans un + discours qui consiste à masquer la 
vérité + je ne suis pas: + dans une forme de complaisance avec le réel j'essaie de le nommer parfois crûment et parfois quand on s'approche de la vérité on s'y brûle

espace de travail d'Emmanuel Macron, son bureau constitue une extension de celui-ci, manifeste symboliquement ses valeurs et démultiplie les indices renvoyant à ses habitudes et à ses méthodes. Un grand tableau à l'effigie de Marianne et avec la devise de la République se trouve à côté de lui, mais le mot le plus saillant, sur le fond bleu blanc rouge, celui que la caméra encadre le plus souvent, c'est le mot «fraternité », ce qui renvoie, une fois de plus, à la dimension éthique de l'eunoia. Les autres vues qui sont régulièrement proposées montrent les drapeaux français et européen, des téléphones et des livres (dont on n'aperçoit cependant pas le titre) posés à côté des dossiers et un peu partout dans la pièce : c'est la culture qui guide l'action politique et pas l'inverse. Macron cite souvent, en effet, des écrivains et des philosophes et aime rappeler qu'il a été l'assistant de Paul Ricœur. La lumière éclatante du lustre et de la pièce, l'apparence lisse des meubles et de la table de marbre autour de laquelle sont assis le président et les journalistes et les rideaux ouverts indiquent enfin la transparence, l'absence de

secrets et de séparation entre le président et les citoyens.

(18) EM : j'assume totalement ce qui a été dit mais que je tiens à faire un distinguo important dans lequel beaucoup de commentateurs + politiques ou journalistiques (j) cela on n'agresse pas ou on n'invective jamais parce que je considère ++ l'ensemble + de mes concitoyens parce que je suis le président de tous les Français

(19) EM : Mais je pense que ce qui est important par rapport à ce que vous évoquiez + surtout cette espèce de + de clivage qu'on pourrait voir entre une France mobile + province + je suis né à Amiens qui est une ville qui a connu beaucoup de difficultés économiques ++ et j'y ai vécu jusqu'à l'- + plus de 16 ans et + l'autre partie de ma famille était dans les Hautes-Pyrénées donc je ne suis pas l'enfant d'une génération spontanée: de nomades itinérants dans un monde où tout va bien

l'éthos montré: celui-ci se manifeste à plusieurs niveaux sémiotiques concourant à la mise en œuvre de la «scénographie » énonciative (Maingueneau 2002), à commencer par la scène matérielle où se déroule l'interview elle-même, à savoir la décoration du bureau, en passant par l'attitude générale de l'orateur et jusqu'à la composante coverbale de sa performance (gestes et prosodie). Dans ce qui suit, nous allons évoquer brièvement le premier point, car les aspects coverbaux demanderaient un article à part (voir, par ailleurs, Druetta 2015). 


\section{L'éthos représenté à travers la sur-énonciation d'un opposant}

Comment les mots de Macron sont-ils repris dans les réactions à son interview ? C'est tout particulièrement un passage de celui-ci qu'on retrouve sous la forme du discours rapporté dans les jours qui suivent l'interview. Il s'agit de la métaphore filée de la " cordée » (la société) et des " premiers de cordée ", que le président de la République a utilisée pour désigner les gens qui réussissent dans les différents domaines, pas uniquement "monétaires ou financiers », comme il s'empresse de le préciser. Reprise dès le lendemain par une centaine d'articles de la presse écrite, comme le montre le tableau réalisé à partir de la banque de données Europresse, et avec un taux de diffusion très élevé au niveau de l'opinion publique, notamment grâce à Twitter, cette expression est devenue emblématique de la vision sociale de Macron.

\section{EVOLUTION}

Peak: 93 documents on October 16, 2017

100

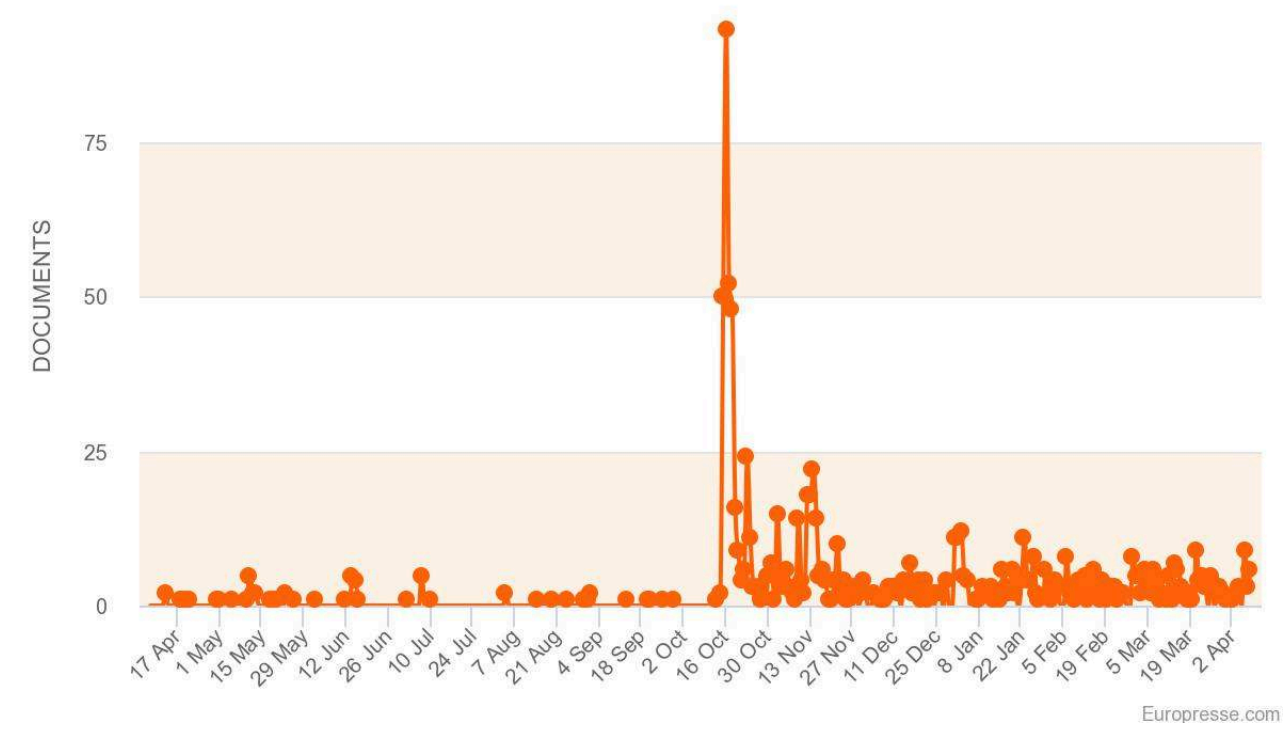

Parmi toutes les réactions, nous avons sélectionné celle de Jean-Luc Mélenchon, lors de l'interview réalisée le lendemain de celle d'E. Macron et diffusée au 20H de France2 par le journaliste Gilles Bouleau (le même qui, la veille, avait interviewé le président), d'une part parce que celui-ci incarne l'un de ses opposants politiques les plus virulents et, d'autre part, parce qu'il a répliqué à la métaphore macronienne en utilisant le même palimpseste verbal, le $\mathrm{SN}$ " premiers de $\mathrm{N}$ », avec une dimension intertextuelle forte, à tel point que cette expression a été reprise et a circulé presque tout autant que celle de Macron. La métaphore « premiers de corvée » surgit en effet à l'intérieur du " résumé » de l'interview présidentielle que Mélenchon livre aux spectateurs en 20 secondes environ. Ce résumé retient essentiellement les aspects considérés comme les plus choquants et a une finalité manifestement ironique. Choisissant l'ironie comme procédé d'argumentation polémique, le locuteur adopte donc une posture de surénonciation surplombante (conformément à Rabatel 2012b) : son résumé contient en même temps un jugement sévère ("vision de caste », «il n'y est pas allé avec le dos de la cuillère », « morgue que je juge insupportable »). Voici la transcription de l'extrait : 
(20) JLM : ce qu'a exprimé euh Emmanuel Macron hier c'est surtout une vision à mon avis de la société qui est une vision de caste et il n'y est pas allé avec le dos de la cuillère + parce que si vous voulez vous étiez témoin direct vous récapitulez alors dans la vie il y a des premiers de cordée c'est ceux [qui ont, qu'ont] des sous il faut pas leur jeter des pierres parce que sinon c'est qu'on est jaloux + et puis il y a les premiers de corvée c'est tous les autres + ils sont au chômage il faut vérifier qu'ils ne sont pas des multi + récidivistes du refus + euh ils luttent à ce moment-là ils deviennent des activistes de la surlégale et puis + même quand il y a le harcèlement, ben c'est dans les quartiers + je trouve qu'une nouvelle fois + il a manifesté cette espèce de morgue + euh + que moi je juge insupportable et je dois pas être le seul.+

41 Le résumé et le jugement sont introduits par une structure pseudo-clivée (« ce qu’a exprimé... ", "c'est une vision... ») et Mélenchon attribue la synthèse à l'énonciateur Gilles Bouleau, son interlocuteur ("vous étiez témoin direct vous récapitulez... »). La synthèse elle-même juxtapose les éléments considérés comme les plus saillants et le locuteur pose des liens de reformulation qui, en sur-énonciation, donnent l'interprétation "authentique » de la parole présidentielle («il y a des premiers de cordée c'est ceux qui ont des sous... il y a les premiers de corvée c'est tous les autres »). Ensuite, la juxtaposition établit des liens hypothétiques paratactiques pour identifier les catégories sociales visées par telle ou telle expression (" s'ils sont au chômage, [alors] il faut vérifier qu'ils ne sont pas des multirécidivistes du refus, s'ils luttent, [alors] ils deviennent des activistes de la surlégale, s'il y a harcèlement, [alors] c'est dans les quartiers »).

Par sa posture surplombante, l'opposant Mélenchon juge la performance de Macron et se place au-dessus de celui-ci, entre autres parce qu'il réussit le coup pragmatique de proposer une étiquette paronomastique qui fait mouche et qui se place sur le même territoire de créativité métaphorique que son adversaire 22 (premiers de cordée vs premiers de corvée). On voit bien que ces données énonciatives - sans être forcément planifiées sont mises au service de la construction de l'éthos ou, tout du moins, cadrent bien avec l'éthos de l'opposant qui, par rapport au pouvoir en place, doit faire preuve d'une moralité inébranlable dans la défense des laissés-pour-compte. Ce sont ici les composantes de la phronesis (sagesse) et de l'areté (vertu dans la vérité) qui sont principalement sollicitées, tandis que l'eunoia peut par endroits leur être sacrifiée, dans la mesure où le locuteur veut s'afficher en position de censeur véhément du pouvoir (c'est ici que la sur-énonciation entre en jeu), et qu'il veut donc se montrer « antipathique » pour certains.

\section{Conclusion : place respective des postures et de l'éthos}

Malgré le caractère momentané, «limité dans l'espace comme dans le temps » (Rabatel, 2017 :78) et aussi « limité à la construction des PDV » (ibid., p. 79), qui fait que les postures "ne sont pas le reflet pur et simple de statuts, de places, de faces » (ibidem), il nous semble qu'il existe des éléments d'affinité entre les dimensions éthotique et posturale, ce qui fonde, par-delà leur autonomie réciproque, la possibilité de les mettre au service l'une de l'autre : un éthos autoritaire semble en effet être en accord avec la posture de sur-énonciation, tandis qu'un éthos déférent (tel celui des journalistes) s'accorde bien avec la sous-énonciation (peu importe à ce stade, qu'il s'agisse d'un signal ou d'un indice). Cet accord se manifeste par un cadre d'attente 
prototypique, déterminé à la fois par l'éthos préalable et les compétences génériques, qui est à la base de la notion de "monde éthique" (Maingueneau 2002). Ce cadre d'attente prototypique se décline différemment pour le destinataire et pour le locuteur. Pour le destinataire, celui-ci aura la forme d'un « crédit de saillance » vis-à-vis de telle posture éventuellement adoptée par le locuteur et, pour ce dernier, celle d'un script énonciatif, sans que cela implique pour autant l'exclusivité d'une seule posture dans toutes les occurrences de discours rapporté, ce que nous avons effectivement constaté dans les différentes prises de parole de Nicolas Hulot, du président ou des journalistes : les tendances dominantes constatées - à savoir la sous-énonciation dans le cas des journalistes, la sur-énonciation en ce qui concerne les responsables politiques n'exclut jamais la possibilité d'avoir recours aux autres postures. Nous avons là la confirmation de la non-systématicité de la relation éthos-posture, en dépit de quelques régularités, comme nous l'avons indiqué supra, $\$ 2$, et, néanmoins, de la pertinence de leur observation conjointe, dès lors qu'un monde éthique constitué vient interposer sa grille de production-interprétation.

Envisagé à partir de la rhétorique, l'éthos apparait comme une dimension dont l'empan est certainement plus large que celui des postures (cf. supra, \$1, points a' et b' notamment). Si, comme nous avons eu l'occasion de le dire, éthos et postures peuvent tous deux s'appuyer sur le verbal et le coverbal, en revanche la gestion de la scénographie et de l'espace matériel d'action, ainsi que la gestion des composantes corporelles coverbales, apparaissent comme davantage liées à l'éthos. Par ailleurs, nous avons souligné le caractère souvent continu, non discrétisable de l'éthos, qui ne se situe pas dans tel ou tel mot, mais plutôt dans l'attitude globale du sujet argumentant, avec une superposition toujours possible entre le locuteur L et le locuteur $\lambda$. Mais, dans la mesure où la posture énonciative est une composante gérée par ce locuteur argumentant, elle a toute sa place dans la constitution de l'éthos - peut-être jusqu'au point d'en faire une marque d'éthos - et nous croyons même que le mérite d'Alain Rabatel est d'avoir permis d'isoler cette composante spécifique, qui aurait pu être mise sur le compte d'autres facteurs plus aléatoires et ne pas être reconnue en tant que telle, avec son poids spécifique dans la construction du discours par rapport au discours d'autrui et à l'interdiscours, ainsi que par rapport aux enjeux pragmatiques manifestés par la rhétorique : la construction de la preuve éthique et l'actio oratoire en particulier. C'est en effet la présence de ces enjeux pragmatiques qui exerce un effet de polarisation généralisée de tous les aspects discursifs et énonciatifs. Tout ce qui concerne la mise en forme du contenu, son actualisation et sa mise en scène pour un auditoire a des retombées argumentatives, et l'on sait bien que la persuasion n'est pas qu'affaire de tête (logos) ou de tripes (pathos), mais que le corps de l'orateur et, plus largement, l'incorporation du discours constituent une composante souvent essentielle de l'argumentation (éthos), depuis le ton de la voix jusqu'aux vêtements, de l'éclairage aux effets de manche, de la manière d'interagir avec des personnes concrètes à la manière de représenter les instances énonciatives autres dans son propre discours, notamment par les positionnements et les postures.

Comme tous les procédés rhétoriques à pivot énonciatif (Bonhomme 2005), la gestion des postures est soumise aux aléas de la réception, qui peut faire tomber à plat les efforts du locuteur, mais il n'en reste pas moins que les traces sont dans le discours et que la fonction de l'analyste est justement de les relever et de les révéler, en montrant 
les réseaux cohérents dans lesquels ils peuvent entrer et les finalités pragmatiques qu'ils peuvent servir.

\section{BIBLIOGRAPHIE}

Amossy, Ruth, 2010, La Présentation de soi. Éthos et identité verbale, Paris, PUF.

Auchlin, Antoine, 2001, «Éthos et expérience du discours : quelques remarques », in M. Wauthion \& A.C. Simon (dir.), Politesse et idéologie. Rencontres de pragmatique et de rhétorique conversationnelle, Louvain, Peeters : 7-95.

Bonhomme, Marc, 2005, Pragmatique des figures du discours, Paris, Honoré Champion.

Brown, Penelope, Stephen C. Levinson, 1987 [1978], Politeness: Some Universals in Language Usage, Cambridge, CUP.

Constantin De Chanay, Hugues, Kerbrat-Orecchioni, Catherine, 2007, « 100 minutes pour convaincre : l'éthos en action de Nicolas Sarkozy ", in M. Broth et al. (dir.), Le français parlé des médias : actes du colloque de Stockholm, 8-12 juin 2005, Stockholm, Université de Stockholm (Acta Universitatis Stockholmiensis) : 309-329.

Druetta, Ruggero, 2015, « La valse à trois temps de la saillance orale : parole, prosodie, geste ", in P. Paissa, F. Rigat et M.-B. Vittoz (dir.), Dans l'amour des mots. Chorale(s) pour Mariagrazia, Alessandria, Dell'Orso : 41-57.

Druetta, Ruggero, à paraître, « Le conflit argumentatif au prisme de la métaphore : l'interview d'E. Macron du 15/10/2017 », in P. Paissa, et al., Métaphore et conflit, Bern, Peter Lang.

Florea, Marie-Laure, 2015, Les Nécrologies dans la presse française contemporaines. Une analyse de discours, thèse de doctorat, Université Lumière Lyon 2.

Maingueneau, Dominique, 2002, « Problèmes d'éthos », Pratiques, 113-114 : 55-67.

Maingueneau, Dominique, 2014, « Retour critique sur l'éthos », Langage et société, 149/3 : 31-48.

Rabatel, Alain, 2004, «Stratégies d'effacement énonciatif et surénonciation dans Le dictionnaire philosophique de Comte-Sponville », Langages, 156 : 18-33.

Rabatel, Alain, 2005a, « Les postures énonciatives dans la co-construction dialogique des points de vue : coénonciation, surénonciation, sousénonciation », in J. Bres, P.P. Haillet, S. Mellet, H. Nølke, L. Rosier (dir.), Dialogisme, polyphonie : approches linguistiques, Bruxelles, Duculot : 95-110.

Rabatel, Alain, 2005b, « Idiolecte et représentation du discours de l'autre dans le discours d'ego », Cahiers de praxématique [En ligne], 44, mis en ligne le 01 janvier 2013, consulté le 26 octobre 2018. URL : http://journals.openedition.org/praxematique/1664

Rabatel, Alain, 2007, « La dialectique du singulier et du social dans les processus de singularisation : style(s), idiolecte, éthos », Pratiques, 135-136 : 15-34.

Rabatel, Alain, 2009, « Prise en charge et imputation, ou la prise en charge à responsabilité limitée », La Notion de prise en charge énonciative, Langue française, 162 : 71-87. 
Rabatel, Alain, 2012a, « Positions, positionnements et postures de l'énonciateur », Travaux neuchâtelois de linguistique, $56: 23-42$.

Rabatel, Alain, 2012b, « Ironie et surénonciation », Vox Romanica, 71 : 1-35.

Rabatel, Alain, 2013, « Humour et sous-énonciation (vs ironie et sur-énonciation) », L'information grammaticale, $137: 36-42$.

Rabatel, Alain, 2014, « Analyse pragma-énonciative des points de vue en confrontation dans les hyperboles vives : hyper-assertion et sur-énonciation ", in L'Hyperbole rhétorique, A. Horak (éd), Travaux neuchâtelois de linguistique, 61-62 : 91-109.

Rabatel, Alain, 2017, Pour une lecture linguistique et critique des médias. Empathie, éthique, point(s) de vue, Limoges, Lambert-Lucas.

Rabatel, Alain, (à paraître), « Éthos dit, éthos montré (ou la présentation de soi représentée, et ce qui s'ensuit en matière de prise en charge et de responsabilité énonciatives). À propos du discours du Bourget de François Hollande, raconté par L. Binet dans Rien ne se passe comme prévu », E-rea : 17-2.

\section{NOTES}

1. Les mots « caractère » " attitude ", « comportement » fournissent autant de synonymes du mot "posture » dans les dictionnaires et sont présents depuis l'Antiquité dans la définition de l'éthos. 2. Dans le couple éthos prédiscursif vs éthos préalable, parfois utilisés comme synonymes, encore que les termes ne se recouvrent pas totalement, nous optons ici pour la notion la plus vaste d'éthos préalable, incluant la "réputation", ainsi que toute sorte d'action accomplie dans la réalité factuelle et extralinguistique. Un bon exemple de retravail de l'éthos préalable par des voies non discursives, nous a été récemment offert par Theresa May, esquissant des pas de danse sur l'air de Dancing Queen, avec une bonne dose d'autodérision, lors de la clôture du Congrès du Parti Conservateur, le 3 octobre 2018.

3. Cf. Maingueneau $2002: 57$.

4. Pour une description de la nature composite et cumulative de l'éthos: cf. Constantin de Chanay et Kerbrat-Orecchioni 2007.

5. Pour une comparaison de ces deux notions, cf. Rabatel 2007.

6. Cf. par exemple, la revendication de Matteo Salvini du 5 mars 2018: "Je suis et je serai toujours fièrement populiste ».

7. Sur la différence entre " prise en charge » et "prise en compte », voir Rabatel 2009 et 2017 : 87-122.

8. Sur la question du nombre indéfini d'ethè possibles et sur le nombre tendanciellement infini des marques à travers lesquelles il se manifeste, nous renvoyons à Auchlin 2001 ; Maingueneau 2014.

9. Notre transcription de l'oral est orthographique, sans majuscules - sauf sur les noms propres et sans ponctuation, à l'exception des guillemets de discours rapporté. Les signes supplémentaires sont: «: " pour marquer les allongements syllabiques; «+» pour marquer la pause (le nombre de «+» étant en rapport avec la longueur de la pause) ; «-» pour marquer l'amorce d'un mot; «( " pour marquer la liaison sans enchaînement (normalement, un $t$ final suivi d'une pause). Les locuteurs politiques sont indiqués par leurs initiales, alors que les journalistes le sont par l'initiale J suivie d'un numéro, pour les distinguer. 
10. Il s'agit d'une phrase attribuée à Bossuet et utilisée comme un argument d'autorité : « Nous sommes d'étranges créatures qui nous affligeons des effets mais qui continuons à adorer les causes. "

11. Ce sont surtout les associations écologistes qui commentent le geste, le qualifiant d'un "gâchis" (Greenpeace) et d'un "électrochoc ", qu'elles espèrent s'avérer salutaire pour le gouvernement (Fondation Nature et Homme).

12. C'est le journal en ligne Contrepoints qui parle de Hulot comme d'un « écologiste autoritaire » et même d'un "idéologue apprenti dictateur»; en revanche, la Fondation Brigitte Bardot le qualifie de «trouillard» (28/8/2018). La possibilité que Hulot ait représenté un "paravent», fonctionnel à une opération de greenwashing du gouvernement, vient par contre de certains milieux écologistes et notamment de François Veillerette. Par ailleurs, pendant l'interview du 28 août, c'est Hulot lui-même qui laisse planer le doute sur l'utilisation à des fins de propagande de son éthos préalable d'activiste et d'animateur de TF1, quand il déclare ne plus vouloir rester, de peur de «donner l'impression par [sa] seule présence » d'être à la hauteur des défis que pose l'écologie.

13. Sur l'antenne de RMC, le 28 août, Benjamin Griveaux a parlé d'une décision qui «manque de courtoisie »; de même, le Ministre de l'Agriculture Stéphane Travert, à cause de son rapport conflictuel avec N. Hulot, s'est senti visé par le réquisitoire fait par le ministre démissionnaire. Cependant, l'attitude de l'entourage gouvernemental a vite tourné à l'éloge du bilan positif des quinze mois du Ministère Hulot.

14. Cf. par exemple, les déclarations du Ministre de l'Action et des Comptes Publics Gérald Darmanin, qui rejette énergiquement l'image de Hulot "avaleur de couleuvres", tout en revendiquant les progrès accomplis pendant son ministère. Cf. aussi l'attitude du successeur de Hulot, François de Rugy qui, dans le discours de passation des pouvoirs du 4 septembre, montre d'abord une attitude de sous-énonciation, lorsqu'il remercie N. Hulot pour son passé d'écologiste engagé et qu'il affirme vouloir s'appuyer sur l'expertise des associations pour la protection de l'environnement; ensuite, il adopte une posture de sur-énonciation, quand il promet de «faire marcher main dans la main économie et écologie ", reprenant ainsi le point le plus sensible et le plus douloureux du discours de Hulot (qui avait affirmé, les larmes aux yeux « je n'ai pas réussi à combler cette ligne de faille entre deux cultures : l'économie et l'écologie »), pour en renverser l'orientation argumentative.

15. Dès sa campagne présidentielle de 2017, on fustige le Président du « en même temps ", un tic de langage dont il a su s'amuser et qu'il a retourné à son avantage (Grand meeting du 17 avril 2017 à Paris-Bercy).

16. Nous faisons bien sûr référence à la théorie des Face Threatening Acts (Brown \& Levinson, 1987). La face positive correspond ici aux dimensions éthotiques de l'eunoia (bienveillance et sympathie) et, de manière secondaire et subordonnée, à la phronesis (modération, sagesse).

17. «Le peuple français ne nous demande pas seulement de l'efficacité. L'efficacité c'est un instrument ! Et puis on peut être tout à fait efficace au service d'une mauvaise cause. Non, il nous demande ce que la philosophe Simone Weil appelait l'effectivité. C'est-à-dire l'application concrète, tangible, visible des principes qui nous guident. Le refus d'être pris en défaut, et de clamer des principes dont nous ne poursuivons pas sans relâche l'application. Le principe d'effectivité, c'est pour vous, pour moi, pour le gouvernement, de ne jamais cesser de se demander si nous sommes en pratique fidèles à nos principes, c'est-à-dire d'abord à la liberté, l'égalité, la fraternité » (3 juillet 2017, discours d'Emmanuel Macron au Congrès).

18. Nous renvoyons à l'article de L. Susini, dans ce même numéro, pour ce qui est de l'emboîtement des PDV, proche de la feintise et néanmoins distinct de celle-ci, par une sorte d'adhésion à la fois au discours cité et à sa propre argumentation, alors que dans la feintise c'est justement l'adhésion au discours cité qui n'est pas sincère. 
19. Ce qui renvoie à la notion de "monde éthique" (Maingueneau 2002: 61): "ce "monde éthique" activé à travers la lecture subsume un certain nombre de situations stéréotypiques associées à des comportements" ".

20. Bien que prototypique, cette posture est loin d'être adoptée par l'ensemble des journalistes dans toutes les situations : pour certains d'entre eux, la sur-énonciation est devenue même leur marque de fabrique (J.-J. Bourdin, J.-M. Aphatie, J.-P. Elkabbach, E. Plenel...), de même, dans les émissions où il $\mathrm{y}$ a plusieurs invités politiques sur le plateau, la fonction de modérateur dévolue au journaliste l'amène souvent à une posture de sur-énonciation: voir à ce sujet l'article d'I. Hekmat dans ce même numéro.

21. Cf. les considérations que Rabatel formule à propos de Nicolas Sarkozy et sur ce qui, chez lui, « fait président » (Rabatel $2017: 20-248$ et 223 -237).

22. À ce sujet, cf. Druetta à paraître.

\section{RÉSUMÉS}

Après avoir comparé, sur un plan théorique, l'empan des notions d'éthos et de posture énonciative, l'article s'interroge sur quelques configurations discursives illustrant la relation de complémentarité qui relie ces concepts. Des situations de crise, de retravail ou de mise en cause de l'éthos (cas de Nicolas Hulot et d'Emmanuel Macron) sont ensuite examinées, afin de décrire : le jeu des ajustements posturaux et des dimensions différentes de l'éthos (dit, montré, représenté) dans la construction éthotique et dans la gestion d'éthos conflictuels; l'articulation du plan énonciatif avec le plan rhétorique et pragmatique; l'interaction avec la scénographie énonciative ; le rapport qui s'établit entre production et réception des postures énonciatives. La question de l'interprétation des postures est enfin analysée dans une situation d'éthos représenté (ironie de Mélenchon à l'égard de Macron).

In this paper first we compare, from a theoretical point of view, the scope of the notions of ethos and enunciative posture, then we discuss some discursive configurations showing how these concepts are possibly shaped by complementarity. Furthermore, we analyse some situations of crisis, reworking and questioning about ethos (Nicolas Hulot's and Emmanuel Macron's cases). The aim is to describe the following: the interrelation between posture tunings and various dimensions of ethos (said, shown, embodied ethos) during ethotic construction and management of contentious ethos; the relationship between enunciative level and pragmatic - rhetorical level; the interaction with enunciative scenography; the connection between production and reception of enunciative postures. The issue of postures' interpretation is finally analysed in a situation of embodied ethos (Melenchon's irony concerning E. Macron).

\section{INDEX}

Keywords : ethos, posture, argumentation

Mots-clés : éthos, posture, argumentation 
AUTEURS

RUGGERO DRUETTA

Università di Torino

PAOLA PAISSA

Università di Torino 